\section{Early detachment of neuromus- cular junction proteins in ALS mice with SODG93A mutation}

\author{
Hisashi Narai,, ${ }^{1,2}$ Yasuhiro Manabe, ${ }^{2}$ \\ Makiko Nagai,' Isao Nagano,' \\ Yasuyuki Ohta, ${ }^{1}$ Tetsuro Murakami, ${ }^{1}$ \\ Yasushi Takehisa, ${ }^{1}$ Tatsushi Kamiya, ${ }^{1}$ \\ Koji Abe' \\ 'Department of Neurology, Graduate \\ School of Medicine, Dentistry and \\ Pharmaceutical Sciences, Okayama \\ University, Okayama, Japan; ' Department \\ of Neurology, National Hospital \\ Organization Okayama Medical Center, \\ Okayama, Japan
}

\section{Abstract}

The transgenic animals with mutant copper/zinc superoxide dismutase (SOD1) DNA develop paralytic motor neuron disease resembling human amyotrophic lateral sclerosis (ALS) patients and are commonly used as models for ALS. In the transgenic (Tg) mice with the G93A mutation of the human SOD1 gene (SOD1 ${ }_{\text {69зA }}$ mice), the loss of ventral root axons and the synapses between the muscles and the motor neurons suggested that the motor neuron degeneration might proceed in a dyingback degeneration pattern. To reveal the relationship between axonal degeneration and the progression of the muscle atrophy in the SOD1 $1_{\text {ตэз }}$ mice, we investigated the status of the neuromuscular junction along the disease progression. As a presynaptic or postsynaptic marker of neuromuscular junction (NMJ), anti-synaptic vesicle protein 2 (anti-SV2) antibody and $\alpha$-bungarotoxin ( $\alpha$-BuTX ) were chosen in this study and, as a marker of synaptic cleft, anti-agrin antibody was chosen in this study. In the immunohistochemistry of $\alpha$ BuTX and anti-SV2 antibody, the percentages of double positive NMJs among $\alpha$-BuTX single positive were decreased in $\mathrm{Tg}$ mice through time from ten weeks. The number of postsynaptic acethylcholine receptor (AChR) clusters did not decrease in $\mathrm{Tg}$ mice even at the end stage. Immunohistochemistry of $\alpha$-BuTX and anti-agrin antibody revealed that the increase of immunopositive area of anti-agrin antibody around the muscle fiber in $\mathrm{Tg}$ mice from ten weeks of age. In this study, we revealed that the detachment of nerve terminals started at ten weeks in Tg mice. The levels of AChR did not change throughout 5-20 weeks of age in both groups of mice, and AChR remains clustering at NMJs, suggesting that the muscle abnormality is the result of detachment of nerve terminals.

\section{Introduction}

Amyotrophic lateral sclerosis (ALS) is a fatal neurodegenerative disease, typically leading to paralysis, respiratory failure and death within 3-5 years after onset. Most patients are sporadic, but about $20 \%$ of familial ALS patients have the mutation in the copper/zinc superoxide dismutase (SOD1) gene, and more than 100 mutations have been reported. ${ }^{1,2}$ The transgenic animals with mutant SOD1 DNA develop paralytic motor neuron disease resembling human ALS patients and are commonly used as models for ALS. ${ }^{3-6}$

Transgenic (Tg) mice with the G93A mutation of the human $S O D 1$ gene (SOD1 $1_{\text {ตэз }}$ mice) were the first established model ${ }^{4}$ and the most widely studied, ${ }^{7}$ so many trials for treatment have used this animal model. The mice showed first clinical signs as a tremor at $80-90$ days after birth, gradually developed paralysis, and died at 140-150 days. In the anterior horn of the spinal cord, the first histological abnormality was mitochondria swelling in motor neuron at 60 days as a consequence of the abnormal stability. ${ }^{8,9}$ Then astrocytosis was revealed around 80 days. Lastly motor neuron loss became prominent from 100 days, when the clinical symptoms were already observed. The axonal transport defects were found as the early events of motor neuron disease,,$^{10-12}$ and the inhibition of retrograde axonal transport by overexpression of dynamitin demonstrated motor neuron degeneration. ${ }^{13}$ Moreover, the loss of ventral root axons and the synapses between the muscles and the motor neurons suggested that the motor neuron degeneration might proceed in a dying-back degeneration pattern. $^{14,15}$ The first event in ALS is the destruction of the neuromuscular junction (NMJ), more specifically of the postsynaptic apparatus, followed by axonal degeneration and late-onset degeneration of motor neuron cell body. ${ }^{14}$ However, recent studies have shown that even a complete rescue of motor neuron cell bodies does not cure mice over-expressing mutant forms of the SOD1 enzyme (mSOD1 mice) ${ }^{16-18}$ Hence, preserving motor neuron cell bodies is therapeutically not sufficient since the rescued motor neurons are unable to recreate destroyed NMJs. To reveal the relationship between axonal degeneration and the progression of the muscle atrophy in the SOD $1_{\text {G93A }}$ mice, we investigated the status of the NMJ along the disease progression.

\section{Materials and Methods}

All experimental procedures were approved by the Animal Care and Use Committee of Okayama University. The SOD1 $1_{\text {g9sa }}$ mice were
Correspondence: Koji Abe, Department of Neurology, Graduate School of Medicine, Dentistry and Pharmaceutical Sciences, Okayama University, 2-5-1 Shikata-cho Kitaku, Okayama 700-8558, Japan. E-mail: abekabek@cc.okayama-u.ac.jp

Key words: amyotrophic lateral sclerosis, neuromuscular junction, $\alpha$-bungarotoxin, SV-2, agrin.

Received for publication: 27 May 2009.

Revision received: 29 September 2009.

Accepted for publication: 29 September 2009.

This work is licensed under a Creative Commons Attribution 3.0 License (by-nc 3.0).

(C) Copyright H. Narai et al., 2009

Licensee PAGEPress, Italy

Neurology International 2009; 1:e16

doi:10.4081/ni.2009.e16

obtained from Jackson Laboratories (B6SJLTgN (SOD1-G93A) 1Gur mice) (Bar Harbor, ME) (Gurney et al., 1994), and the line was maintained as hemizygotes by mating SOD1 $1_{693 \mathrm{~A}}$ males with $\mathrm{C} 57 \mathrm{BL} / 6 \mathrm{~J}$ females. The offspring were genotyped by a polymerase chain reaction assay of DNA obtained from tail tissue. They are the high-expressing G93A SOD1 mutant mice that display disease onset at 15 weeks and die approximately four weeks later. Since there is a difference in disease onset between male and female SOD1 $1_{\text {G9за }}$ mice, only males were used in this study.

We analyzed disease progressions by a rotarod task every seven days. We measured the time until mice fell from the rod or after an arbitrary limit of 600 seconds. We performed three trials, and recorded the average time for every mouse. For histological studies and Western blot analysis, SOD1 $1_{\text {g93 }}$ mice and nontransgenic mice were deeply anesthetized at five, ten, 15 and 20 weeks of age (each group $\mathrm{n}=5$ ), and transcardially perfused with heparinized phosphate-buffered saline (PBS) (pH 7.4). The right soleus muscles were frozen for Western blot examination, and the left side muscles were quickly frozen by liquid nitrogen for histological studies. The right side muscles were cut on cryostat at a thickness of $10 \mu \mathrm{m}$ at $-20^{\circ} \mathrm{C}$. Frozen transverse sections were fixed in 4\% paraformaldehyde for $15 \mathrm{~min}$, and stained with hematoxylin and eosin (HE) for morphological assessment. For immunohistochemistry, the sections were incubated with $5 \%$ bovine serum albumin for one hour at room temperature to block the non-specific binding of antibodies. Sections were reacted with primary antibodies overnight at $4^{\circ} \mathrm{C}$. The following primary antibodies were used: anti-SV2 (sc-11936, Santa Cruz Biotechnology, CA, USA), anti-agrin (MAB5204, Chemicon, Temecula, CA, USA), and $\alpha$-bungarotoxin (BuTX) biotin-XX-conjugate (B-1196, Molecular 
Probes, Eugene, OR, USA). After washing with PBS, the sections were exposed to appropriate secondary antibodies conjugated fluorescent dye for one hour at room temperature, then washed with PBS again, and mounted. A set of sections was treated without the primary antibody to ascertain the specific detection by the antibodies used. Sections were examined and photographed through a microscope (Olympus BX 51) under epifluorescent illumination. We evaluated the remaining neuromuscular junctions (NMJs) as counting $\alpha$-BuTX and SV2double positive synapses with a diameter above $20 \mu \mathrm{m}$. Counting was performed by investigators blinded to the genotype. Multiple group comparisons of the differences in quantitative measurements were made by Student's t-test. Statistical significance was accepted at the level of $p<0.05$. The protein level for acethylcholine receptor (AChR) was analyzed by SDS-PAGE and transferred to a PVDF membrane (Millipore Corporation, Bedford, MA, USA). The blots were blocked in 5\% dry milk in Tris-buffered saline for one hour at room temperature. The proteins were detected by incubating the blots overnight at $4^{\circ} \mathrm{C}$ with the first primary antibodies, followed by HRP-conjugated secondary antibody (GE Healthcare Bio-sciences Corp., Piscataway, NJ, USA) in blocking solution for one hour at room temperature. After washing, the blot was visualized with enhanced chemiluminescent detection kit (Vector) and exposed to X-ray films (AgfaGevaert N.V., Belgium). Densitometric evaluation of reactive protein bands was performed using Scion imaging software. The membrane was washed by stripping buffer, then $\beta$-tubulin (T-4026, Sigma Aldrich co., St. Louis, MO, USA) used as an internal control.

\section{Results}

Clinical study showed that SOD1 ${ }_{693 \mathrm{~A}}$ mice (Tg mice) revealed foot tremor from about 15 weeks of age and the rotarod score significantly decreased at $18 \quad\left({ }^{*} p<0.05\right)$ and 19 $\left({ }^{* *} p<0.01\right)$ weeks of age, and died around 20 weeks (Figure 1). Histological findings of soleus muscles with HE staining showed that neurogenic muscular fiber atrophy was already present at ten weeks of age and became severe with time from 15 and 20 weeks in $\mathrm{Tg}$ mice (Figure 2, lower panels).

As a presynaptic or postsynaptic marker of NMJ, anti-SV2 antibody and $\alpha$-BuTX were chosen in this study, which detects synaptic vesicles of axon terminals and AChR of muscles, respectively. As a marker of synaptic cleft, antiagrin antibody was chosen in this study, which detects anchoring proteins of NMJs. We estimated functional NMJs with double positive for both pre- and postsynaptic markers (Figure

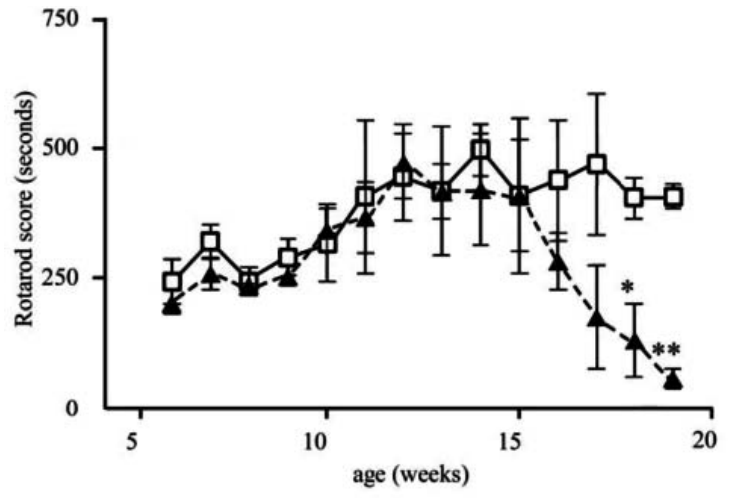

Figure 1. Clinical scores of rotarod tests. Note the decrease of the score after 15 weeks in Tg mice (filled triangles) as compared to wild-type (open squares) $\left({ }^{*} p<0.05,{ }^{* *} p<0.01\right)$.

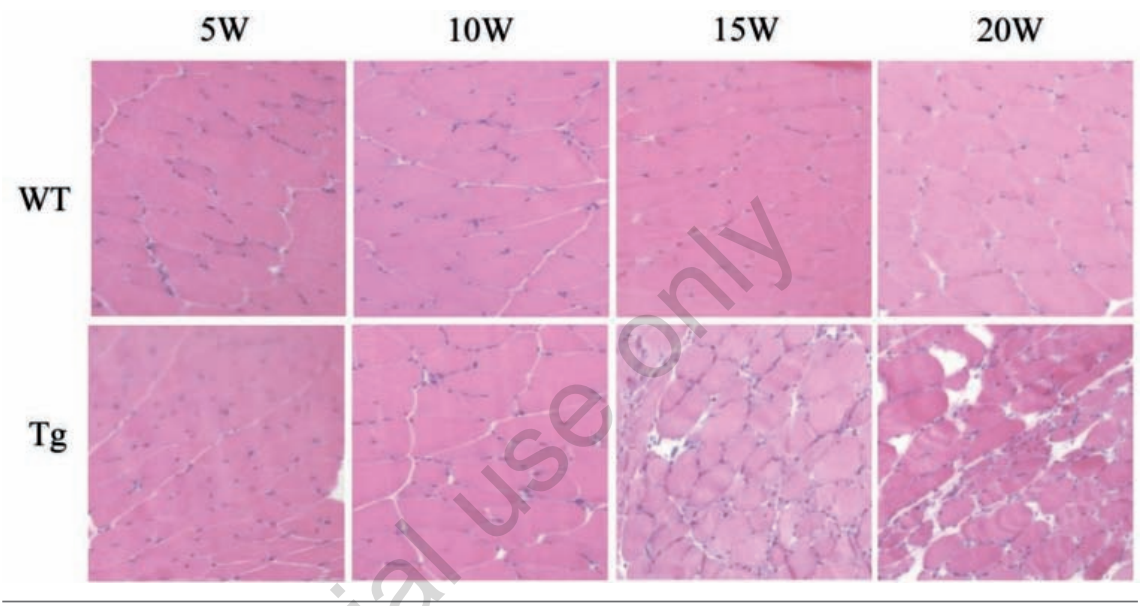

Figure 2. HE staining of soleus muscles in wild-type (WT) and Tg mice. Note a subtle change in 10 weeks of $\mathrm{Tg}$ mice, with deteriorating 15-20 weeks of age.
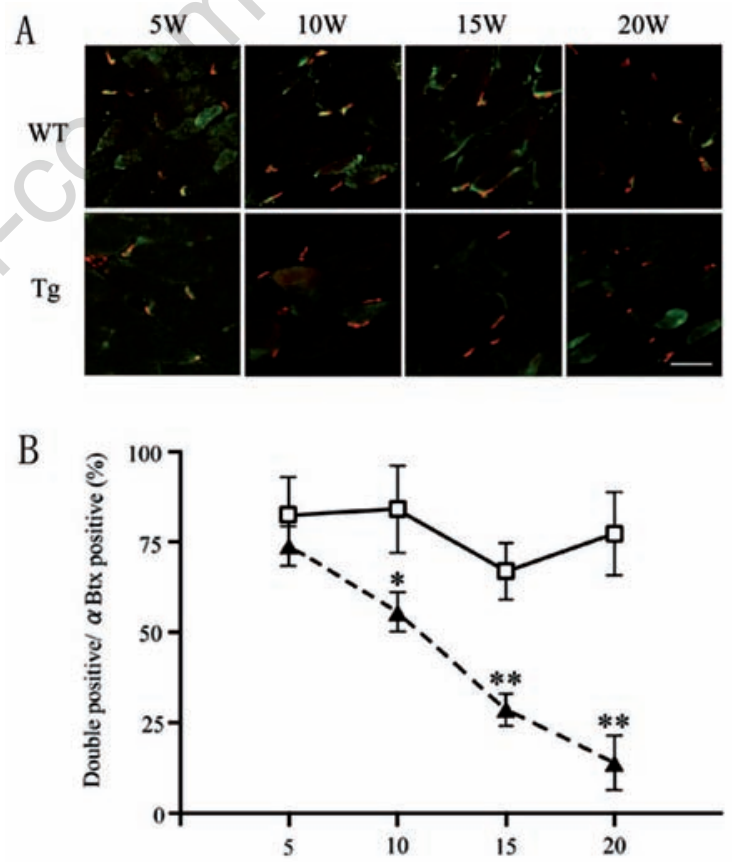

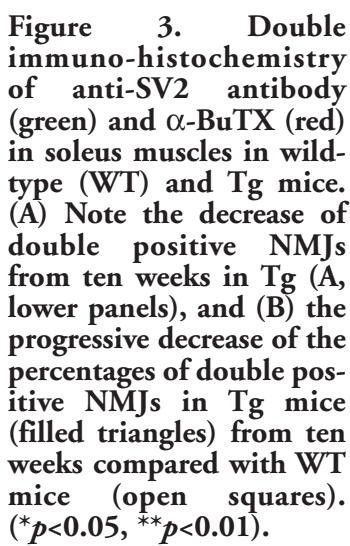

$3 \mathrm{~A})$. The percentages of double positive NMJs among $\alpha$-BuTX single positive were not significantly different between WT and Tg mice at five weeks $(82.2 \pm 9.3 \%$ and $67.2 \pm 16.0 \%$ respec- tively), which then decreased in $\mathrm{Tg}$ mice through the time from ten weeks $(62.5 \pm 13.8 \%$ at ten weeks, $28.7 \pm 8.8 \%$ at 15 weeks, $13.9 \pm 10.4 \%$ at 20 weeks) (Figure $3 \mathrm{~B}$, filled tri- 
angles). The number of postsynaptic AChR clusters did not decrease in $\mathrm{Tg}$ mice even at the end stage (20 weeks). Immunohistochemistry of $\alpha$-BuTX and anti-agrin antibody revealed that there was no increase of the expression levels of agrin throughout the time course in the control muscle, and the immunopositive area of anti-agrin antibody almost co-localized with the immunopositive area of $\alpha$-BuTX. However, we found the increase of immunopositive area of anti-agrin antibody around the muscle fiber in $\mathrm{Tg}$ mice from ten weeks of age. (Figure 4A). In Western blot analysis, the intensity level of $\alpha$-BuTX did not significantly differ between wild-type and $\mathrm{Tg}$ mice throughout 5-20 weeks of age (Figure 4B and $\mathrm{C}$ ).

\section{Discussion}

In this study, we revealed that the detachment of nerve terminals started at ten weeks, which is still asymptomatic stage of the disease course in $\mathrm{Tg}$ mice. At ten weeks of age, Tg mice looked normal and showed the same scores as wild-type mice in rotarod test, thus histological abnormality in soleus muscles preceded the clinical signs. Muscle fiber changes also started at ten weeks in Tg mice. Muscle atrophy progressed with age (Figure 2) along with the loss of functional NMJs before motor neurons remained in the spinal cord. These data suggest that a part of spinal motor neurons may lose their function with territorial muscle fibers even though the neurons showed normal morphologies. With close analysis of NMJ proteins, the SV2 that is the presynaptic marker of the NMJs decreased with disease progression (Figure 3). Decrease of the SV2 starts at ten weeks of age, indicating that nerve terminals detach from NMJs from the time of ten weeks of age of Tg mice. Agrin is a large extracellular matrix proteoglycan, which potentiates the clustering AChR by interacting with several postsynaptic molecules, such as dystroglycan, integrins, and muscle-specific tyrosine kinase (MuSK) and organizes the postsynaptic differentiation. ${ }^{19}$ There are alternative splicing isoforms of agrin, derived from motoneurons or muscles. ${ }^{20}$ The immunoreactivities of agrin in the muscle spaces developed stronger with the disease progression of $\mathrm{Tg}$ mice (Figure 4). Muscle agrin plays an important role in preserving the transverse orientation in denervated muscles, though neural agrin more actively aggregates the AChR. ${ }^{21}$ To our surprise, agrin expression at NMJs did not decrease in atrophic muscles in $\mathrm{Tg}$ mice, but increased at 20 weeks of age (Figure 4B). Because the present antibody recognized both nerve- and musclederived agrins, the expression levels of muscle agrin may increase during the disease progres-
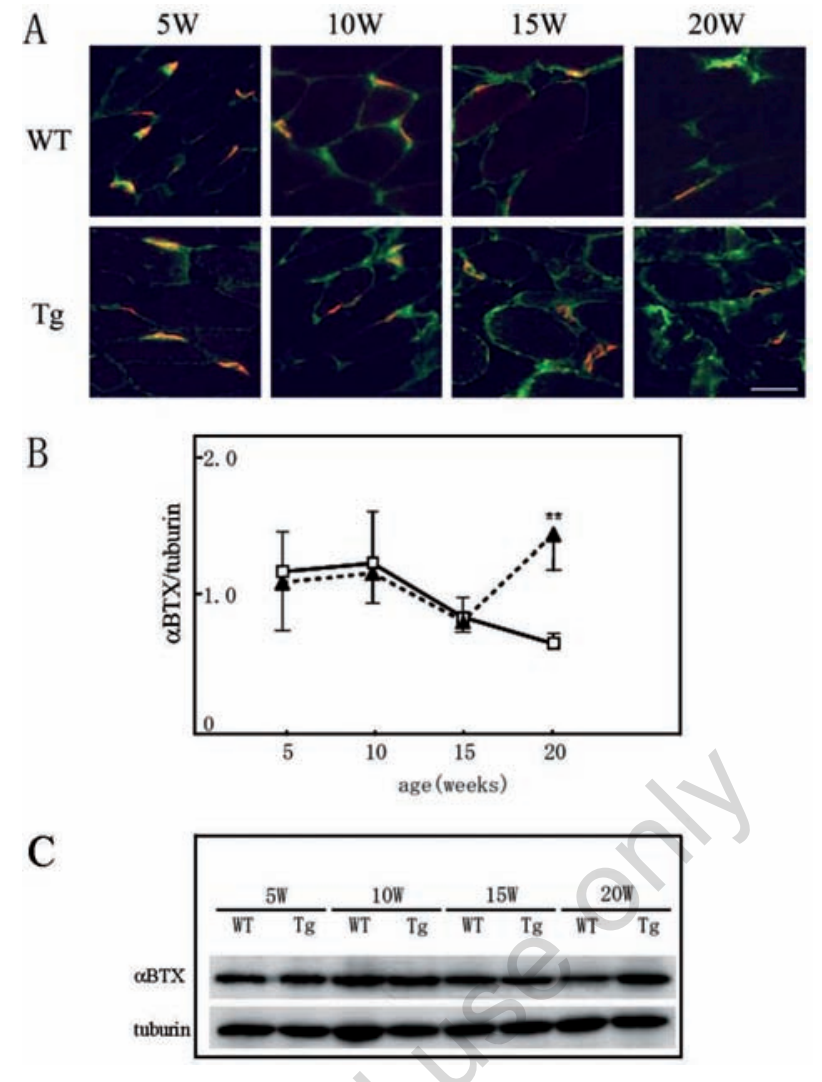

Figure 4. Double immunohistochemistry of agrin (green) and $\alpha$-BuTX (red) (A and $B$ ), and Western blotting of AChR of soleus muscles (C). Note the increase of agrin in the muscle spaces at 20 weeks in Tg mice (A, B; filled triangles), while no change in $\alpha$-BuTX between WT and Tg mice in Western blot (C).

sion in Tg mice. The levels of AChR did not change throughout 5-20 weeks of age in both groups of mice, and AChR remains clustering at NMJs (Figure 4), suggesting that the muscle abnormality is the result of detachment of nerve terminals.

A previous report showed that an axotomy resulted in the nerve terminal degeneration, where AChR remained clustering at the original sites..$^{15}$ Subsequently, regenerating axons differentiated into nerve terminals when they contacted original synaptic basal lamina. ${ }^{15}$ The detachment characteristics in the current $\mathrm{Tg}$ mice were similar to such a distal axonopathy ${ }^{14,15}$ but the regeneration and the recomposition of the functional NMJs never happened in the current $\mathrm{Tg}$ mice. This difference may be profoundly related to the pathogenesis of this ALS model mice, which could be explained by the defect of axonal transport in Tg mice. ${ }^{10}$

\section{References}

1. Brown RH Jr. Amyotrophic lateral sclerosis: recent insights from genetics and transgenic mice. Cell 1995;80:687-92.

2. Pasinelli P, Brown RH. Molecular biology of amyotrophic lateral sclerosis: insights from genetics. Nat Rev Neurosci 2006; 7:710-23.

3. Bruijn LI, Becher MW, Lee MK, et al. ALS- linked S0D1 mutant G85R mediates damage to astrocytes and promotes rapidly progressive disease with SOD1-containing inclusions. Neuron 1997;18:327-38.

4. Gurney ME, Pu H, Chiu AY, et al. Motor neuron degeneration in mice that express a human $\mathrm{Cu}, \mathrm{Zn}$ superoxide dismutase mutation. Science 1994;264:1772-5.

5. Nagai M, Aoki M, Miyoshi I, et al. Rats expressing human cytosolic copper-zinc superoxide dismutase transgenes with amyotrophic lateral sclerosis: associated mutations develop motor neuron disease. J Neurosci 2001;21:9246-54.

6. Wong PC, Pardo CA, Borchelt DR, et al. An adverse property of a familial ALS-linked SOD1 mutation causes motor neuron disease characterized by vacuolar degeneration of mitochondria. Neuron 1995;14: 1105-16.

7. Bendotti C, Carri MT. Lessons from models of SOD1-linked familial ALS. Trends Mol Med 2004;10:393-400.

8. Bendotti C, Calvaresi N, Chiveri L, et al. Early vacuolization and mitochondrial damage in motor neurons of FALS mice are not associated with apoptosis or with changes in cytochrome oxidase histochemical reactivity. J Neurol Sci 2001;191: 25-33.

9. Bergemalm D, Jonsson PA, Graffmo KS, et al. Overloading of stable and exclusion of unstable human superoxide dismutase-1 variants in mitochondria of murine amy- 
otrophic lateral sclerosis models. J Neurosci 2006;26:4147-54.

10. Warita H, Itoyama Y, Abe K. Selective impairment of fast anterograde axonal transport in the peripheral nerves of asymptomatic transgenic mice with a G93A mutant SOD1 gene. Brain Res 1999; 819:120-31.

11. Williamson TL, Cleveland DW. Slowing of axonal transport is a very early event in the toxicity of ALS-linked SOD1 mutants to motor neurons. Nat Neurosci 1999;2:50-6.

12. Zhang B, Tu P, Abtahian F, et al. Neurofilaments and orthograde transport are reduced in ventral root axons of transgenic mice that express human SOD1 with a G93A mutation. J Cell Biol 1997;139:130715.

13. LaMonte BH, Wallace KE, Holloway BA, et al. Disruption of dynein/dynactin inhibits axonal transport in motor neurons causing late-onset progressive degeneration. Neuron 2002;34:715-27.

14. Fischer LR, Culver DG, Tennant P, et al. Amyotrophic lateral sclerosis is a distal axonopathy: evidence in mice and man. Exp Neurol 2004;185:232-40.

15. Pun S, Santos AF, Saxena S, et al. Selective vulnerability and pruning of phasic motoneuron axons in motoneuron disease alleviated by CNTF. Nat Neurosci 2006;9: 408-19.

16. Gould TW, Buss RR, Vinsant S, et al. Complete dissociation of motor neuron death from motor dysfunction by Bax deletion in a mouse model of ALS. J Neurosci 2006;26:8774-86.

17. Rouaux C, Panteleeva I, René F, et al. Sodium valproate exerts neuroprotective effects in vivo through CREB-binding protein-dependent mechanisms but does not improve survival in an amyotrophic lateral sclerosis mouse model. J Neurosci 2007; 27:5535-45.

18. Dupuis L, Loeffler J-P. Neuromuscular junction destruction during amyotrophic lateral sclerosis: insights from transgenic models. Curr Opin Pharmacol 2009;9:341-6.

19. Sanes JR, Lichtman JW. Induction, assembly, maturation and maintenance of a postsynaptic apparatus. Nat Rev Neurosci 2001;2:791-805.

20. Burgess RW, Nguyen QT, Son YJ, et al. Alternatively spliced isoforms of nerveand muscle-derived agrin: their roles at the neuromuscular junction. Neuron 1999; 23:33-44.

21. Bezakova G, Lomo T. Muscle activity and muscle agrin regulate the organization of cytoskeletal proteins and attached acetylcholine receptor (AChR) aggregates in skeletal muscle fibers. J Cell Biol 2001;153: 1453-63. 